\title{
The frequency range of TMJ sounds
}

\author{
S. E. WIDMALM*, W. J. WILliAMS ${ }^{\dagger}$, D. DJURDJANOVIC ${ }^{\ddagger}$ \& D. C. MCKAY ${ }^{\star}{ }^{*}$ Depart- \\ ments of Biologic and Materials Sciences, School of, Dentistry and of Electrical Engineering and Computer Science, College of Engineering, \\ University of Michigan, Ann Arbor, MI, U.S.A., ' Department of Electrical Engineering and Computer Science, College of Engineering, \\ University of Michigan, Ann Arbor, MI, U.S.A., ${ }^{\ddagger}$ Department of Mechanical Engineering, University of Michigan, Ann Arbor, MI, USA and \\ \$9201 Sunset Blvd, Suite 912, Los Angeles, CA, U.S.A.
}

SUMMARY There are conflicting opinions about the frequency range of temporomandibular joint (TMJ) sounds. Some authors claim that the upper limit is about $650 \mathrm{~Hz}$. The aim was to test the hypothesis that TMJ sounds may contain frequencies well above $650 \mathrm{~Hz}$ but that significant amounts of their energy are lost if the vibrations are recorded using contact sensors and/or travel far through the head tissues. Time-frequency distributions of 172 TMJ clickings (three subjects) were compared between recordings with one microphone in the ear canal and a skin contact transducer above the clicking joint and between recordings from two microphones, one in each ear canal. The energy peaks of the clickings recorded with a microphone in the ear canal on the clicking side were often well above $650 \mathrm{~Hz}$ and always in a significantly higher area (range $117-1922 \mathrm{~Hz}, P<0.05$ or lower) than in recordings obtained with contact sensors (range 47-375 Hz) or in microphone recordings from the opposite ear canal (range 141-703 Hz). Future studies are required to establish normative frequency range values of TMJ sounds but need methods also capable of recording the high frequency vibrations. KEYWORDS: temporomandibular joint, clicking, sound, Fourier transform, time-frequency distribution, power spectral density, signal processing, frequency range

\section{Introduction}

Temporomandibular joint (TMJ) sounds are important signs of temporomandibular disorder (TMD) and routinely recorded in patients with suspected TMD, but verbal descriptions of sounds* recorded by auscultation are not suited for comparisons between observers (Watt, 1963, 1966). Many attempts have therefore been made to instead record TMJ sounds electronically when collecting such data for statistical analysis (Ekensten, 1952; Watt, 1963, 1966; Ouellette, 1974; Widmalm \& Hedegård, 1974; Gay \& Bertolami, 1987; Hutta et al., 1987; Prinz, 1998). Different types of sensors have been used, microphones (Watt, 1963, 1966; Widmalm, Williams \& Adams, 1996b; Ouellette,

*The term sound refers to vibrations in the audible range, 20$20000 \mathrm{~Hz}$, subsonic sounds refer to vibrations $<20 \mathrm{~Hz}$, ultrasonic sounds refer to vibrations above $20000 \mathrm{~Hz}$. The term vibration can be used for all types of sounds.
1974; Prinz, 1998; Leader et al., 1999), accelerometers (Owen, 1996; Widmalm \& Hedegård, 1974; Ishigaki, Bessette \& Maruyama, 1993), or a combination of microphone and stethoscope (Watt, 1966; Gallo et al., 1993). The sites chosen for placing the microphone have also differed between authors. Some have recorded with the microphone in contact with the skin above the zygomatic arch (Ouellette, 1974). Others placed the microphone in the auditory meatus (Yoshida et al., 1994; Widmalm et al., 1996b; Sano et al., 1999).

The design of a system for electronic recording and analysis of TMJ sounds is dependant on what frequency area the research is aimed to study. The results are therefore significantly affected by the choice of sampling rate (SR). The methods used for electronic recording of TMJ sounds differ significantly in this aspect. Presently some authors claim that little if any energy is to be found in the frequency area above about $650 \mathrm{~Hz}$. (Ishigaki et al., 1993; Owen, 1996; Olivieri et al., 1999; 
Garcia et al., 2000) and restrict the SR to $1300 \mathrm{~Hz}$ or lower. Other authors report that they have recorded frequencies well above $650 \mathrm{~Hz}$ (Widmalm, Williams \& Zheng, 1991; Yoshida et al., 1994; Prinz, 1998; Sano et al., 1999) and Widmalm et al. (1996b) recommended an SR of at least about $10000 \mathrm{~Hz}$.

The question about the true frequency range of TMJ sounds and the optimal SR can be brought closer to a solution by recording the same TMJ sounds with more than one type of sensor. More accurate comparisons can then be made between the characteristics, such as time-frequency distributions and power spectra, of the TMJ sounds recorded with different types of sensors and sites (Fig. 1) for attachment. There is a need for such studies to facilitate optimal configuration of hardand software in future studies on TMJ sounds.

The hypotheses were

- That TMJ sounds may contain frequencies well above $650 \mathrm{~Hz}$.

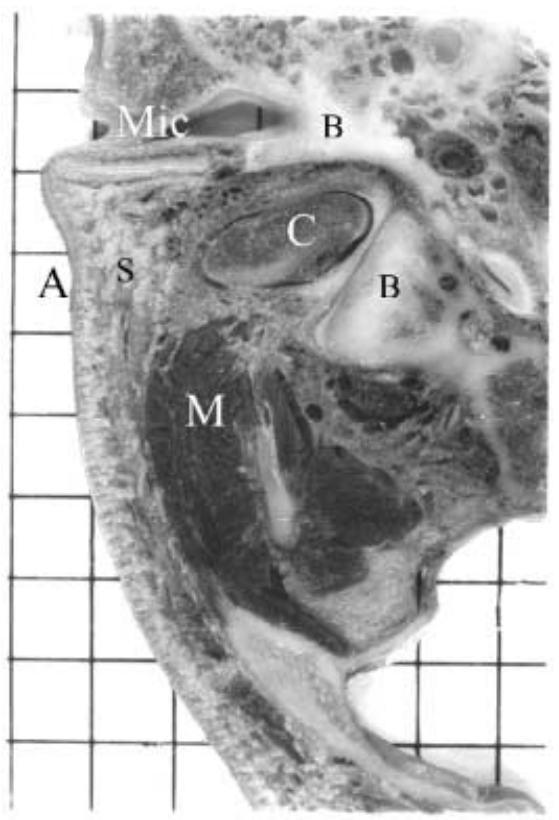

Fig. 1. Horizontal section through the condylar (C) area. This figure illustrates how the pathways may differ when the sounds propagate through bone (B) and soft (S) tissues to different sites that may be chosen for placement of the transducer. The microphone site (Mic) can be in the auditory meatus very close to the joint where the sound originates. An accelerometer is usually placed in contact with the skin at a site (A) where the thickness of the soft tissues is larger than between the TMJ and the auditory meatus and may vary significantly between patients. $\mathrm{M}=$ masseter muscle.
- That the TMJ sounds are reduced in energy content when they propagate through head tissues.

- That the high frequency parts of TMJ sounds above $650 \mathrm{~Hz}$ are more reduced in energy content in recordings with skin contact transducers than in microphone recordings.

The aim was

- To compare the frequency content of TMJ vibrations recorded simultaneously with a microphone at the opening of the ear canal and with transducers placed with skin contact above the same side TMJ and with a microphone at the contralateral ear opening.

\section{Methods}

Two-channel TMJ sound recordings $(n=172)$ were made from three adult subjects (two females, one male) who had non-painful clicking. The subjects were fully informed of the experiments and agreed to take part.

The TMJ clickings were recorded with two small electret condenser microphones (diameter $5 \mathrm{~mm})^{\dagger}$, bandwidth 40-20 000 Hz and with a piezoelectric accelerometer $^{\ddagger}$ that had a resonant frequency at $3800 \mathrm{~Hz}$ and a flat response between 30 and $3600 \mathrm{~Hz}$. Electronic recordings were also made with a microphone placed in the earplug of a stethoscope with $50 \mathrm{~cm}$ long tubing while the diaphragm of the endpiece was held in contact with the skin above the TMJ. The stethoscope was of a commonly used type as illustrated in Jarvis (1992).

A headphone holder held the accelerometer in place over the lateral aspect of TMJ. The microphones and the stethoscope were held in place manually.

The microphones were calibrated at the start of each session by recording a $1000-\mathrm{Hz}$, signal ${ }^{\S}$ that had a 94$\mathrm{dB}$ (RMS) peak-to-peak sound pressure level ${ }^{\text {II }}$ (peSPL) (Mendel, Danhauer \& Singh, 1999). The signals were

\footnotetext{
†Sony ECM-77B, Sony Corporation, 6-7-35, Kita-shinagawa, Shinagawa-ku, Tokyo 141, Japan.

${ }^{\ddagger}$ Model 207 A, Bioresearch, Milwaukee, WI, U.S.A.

§Sound Level Calibrator Type 4231, Brüel \& Kjær, A/S, Nærum, Denmark.

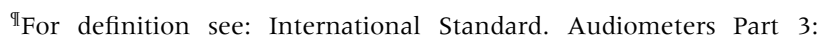
Auditory test signals of short duration for audiometric and neuro-otological purposes. Reference number CEI/IEC 645-3, pp. 1-18 (1994).
} 

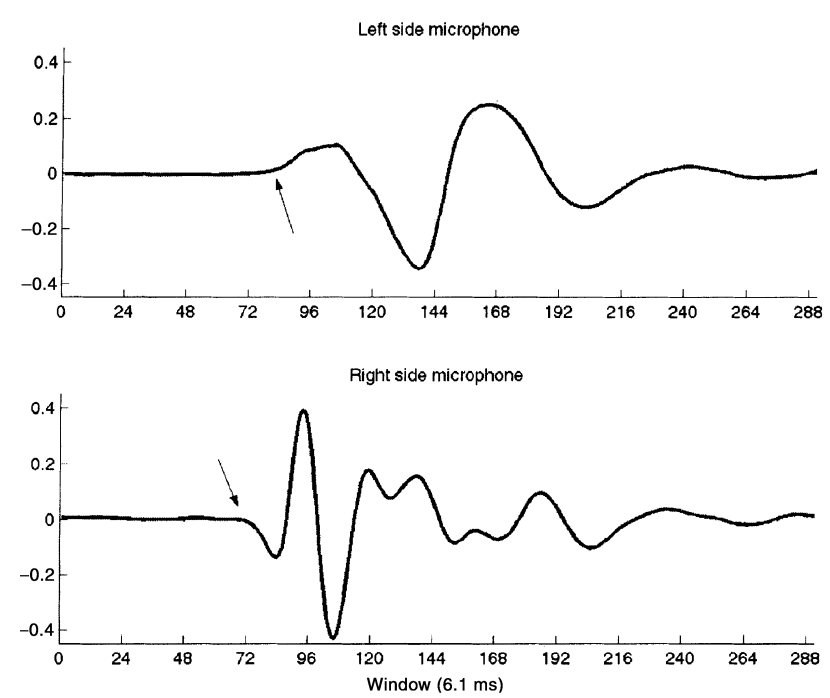

Fig. 2. The localization of a clicking to side was made by observing the time difference between bilateral recordings of the same sound. The recordings were made with one microphone in the left (upper window) and one in the right (lower window) ear canal. Arrows mark where the potentials start to deviate from the baseline. The sound recorded with the right side microphone starts about $0 \cdot 2 \mathrm{~ms}$ earlier than the one in the upper window recorded with the left side microphone. The conclusion is that the sound came from the right joint. The waveform in the upper window is more 'smooth' as a result of filtering of the sound signal while passing a longer distance through head tissues than the sound in the lower window had to. Horizontal axes are in samples. Sampling rate was $48000 \mathrm{~Hz}$. Vertical axes are in parts of the A/D card's dynamic range $(1=$ maximal positive and $-1=$ maxmaximal negative values).

digitized using a 24-bit sound card $^{* *}$ and an SR of either 48000 or $96000 \mathrm{~Hz}$. Most clickings can be recorded both on the side of origin and on the contralateral side (Watt, 1966; Hashimoto et al., 1990; Widmalm, Williams \& McKay, 2002). The contralaterally recorded sound (here called an 'echo'), has most often, but not always, a slightly lower peSPL. We, therefore, decided the side of origin by comparing the time locations because the echo appears about $1 \mathrm{~ms}$ or less later in time (Widmalm et al., 2002) as illustrated in Fig. 2. The transfer function of the clicking sounds was calculated as described by Widmalm et al. (2002) to ensure that each pair of sensors was recording the same sound. Magnitude, phase shift and time delay were compared between the two groups of sound recordings made during each patient session. They were accepted as

**Direct Pro 24/96. Aardvark, Ann Arbor, MI, U.S.A. being from the same clicking if the coherence was $\geq 80 \%$.

The recordings were made in a quiet office. With the computer (PC) on, the noise level was about $55 \mathrm{~dB}$ peSPL (RMS) as measured ${ }^{+\dagger}$ (C-weighting) $1 \mathrm{~m}$ from the PC (Mendel et al., 1999). The C-weighting is essentially flat. A-weighting that would have given much lower $\mathrm{dB}$ values is based on the 40-phon Fletcher-Munson equalloudness contour (Ballou, 1998) and was not adequate here because the electronic TMJ sound recordings were not adjusted that way. To the best of our knowledge such adjustments have not been used in TMJ sound research. With the PC off the level was close to $50 \mathrm{~dB}$ peSPL. Test recordings were made with all sensors during mandibular rest. The energy content in those recordings was negligible when compared with the energy content in the TMJ clicking sounds. The equivalent noise peSPL during jaw movements and between clickings or crepitations was negligible in linear graphical displays of the power spectra.

The recordings were always made with one microphone at the ear canal opening on the same side as the clicking joint. Recordings were made in five sessions with the second transducer placed as follows.

Session 1. An accelerometer held by a headphone frame in contact with the skin above the same side TMJ.

Session 2. A second microphone mounted in the earplug of a stethoscope with the stethoscope's endpiece held in contact with the skin above the same side TMJ.

Session 3. A second microphone held at the opening of the contralateral ear canal.

Session 4. A second microphone held about $2 \mathrm{~mm}$ above the skin above the same side TMJ.

Session 5. A second microphone held in contact with the skin above the same side TMJ.

The TMJ sounds were recorded during jaw openingclosing cycles (about 1-2 cycles per second). The subjects were asked to open as wide as they could, at least 10, and, if possible without feeling pain or discomfort, 15-25 times. Sessions 4 and 5 were not repeated with subject nos 2 and 3 because they started to feel slight discomfort after finishing session no. 3.

The analogue waveforms were continuously displayed on the PC monitor during the opening-closing

${ }^{+\dagger}$ Radio Shack Sound Level Meter, Tandy Corporation, Fort Worth, TX, U.S.A. 
cycles to check that adequate gain was used. The recordings were analysed using Cool Edit Pro $^{\ddagger \ddagger}$, Matlab $^{\text {} \$}$ software, and application programs written in Matlab. The locations of the energy peaks of the clicking sounds in the frequency domain were estimated from the binomial kernel Reduced Interference TimeFrequency Distributions (Williams \& Jeong, 1991; Jeong \& Williams, 1992), some of which are shown as contour plots in Fig. 3.

Power spectra (Figs 4a, 5a and 6a) were produced to illustrate differences in the distribution of energy in relation to frequency. Cumulative energy distributions were also plotted where the percentage of energy below and above a certain frequency could be read (Figs $4 \mathrm{~b}$, $5 \mathrm{~b}$ and $6 \mathrm{~b}$ ). The signals were stored with lengths, long enough to contain the total duration of the sound waveform of interest, if possible without including neighbour sounds. The power spectral density (PSD) was calculated using Welch's periodogram method (Welch, 1967) as embodied by the Matlab function 'SPECTRUM'. The length of the Fourier Transform (NFFT) was the same as, or slightly longer than, the length of the signal and always a power of 2 . The power of a signal over a particular frequency band was found by summing the PSD over that band.

The energy peak locations were compared within subjects and between sensors using Paired Samples $t$-test and ANova General Linear Model for Repeated Measures. ${ }^{\text {III }}$ The null hypothesis that the samples came from populations with the same variance was tested using the Levene test (Norušis, 2000).

\section{Results (details are given in Tables 1 and 2)}

The energy peaks were always in a significantly higher part $(P<0.05$ or less, Table 1$)$ of the frequency domain in the recordings with the microphone at the opening of the auditory canal than with the microphone mounted in a stethoscope or in the recordings with any of the sensors in contact with the skin above the same side TMJ. The peaks were also in a significantly lower frequency area $(P<0.01$ or less, Table 1) in the recordings with a second microphone in the ear canal on the side opposite to the clicking joint.

\footnotetext{
${ }^{\ddagger \ddagger}$ Syntrillium Software Corporation, Phoenix, AZ, U.S.A.

${ }^{\S}$ The MathWorks Inc., Natick, MA, U.S.A.

${ }^{\text {IIII }}$ SPSS V10・0, SPSS, Chicago, IL, U.S.A.
}
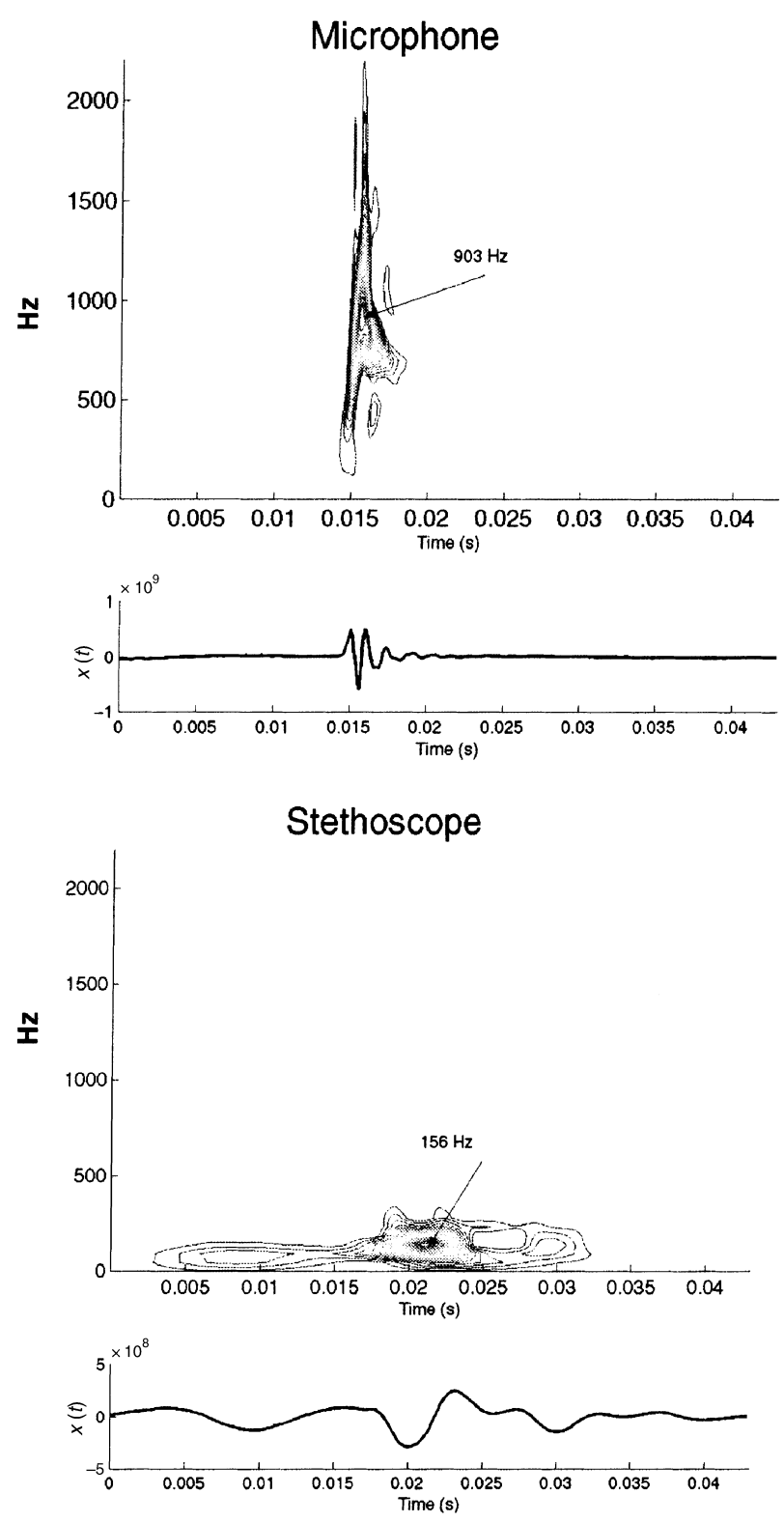

Fig. 3. Contour plots in this figure illustrate the differences in the time and frequency location of the energy peaks in binomial kernel reduced interference distributions of the same clicking recorded with a microphone in the ear canal and with a microphone placed in the earplug of a stethoscope. The end-piece of the stethoscope was held in contact with the skin above the TMJ. The energy peak in the stethoscope recording $(156 \mathrm{~Hz})$ is in a much lower frequency area than in the microphone recording (903 Hz) and also delayed in time, because of the longer path over which the sound has to pass through the stethoscope tubing, as compared with the recording with the microphone in close proximity to the TMJ. The smaller windows under the contour plots show the respective analogue waveforms. There the vertical axes are in parts of the A/D card's dynamic range $(1=$ maximal positive and $-1=$ maximal negative values). 

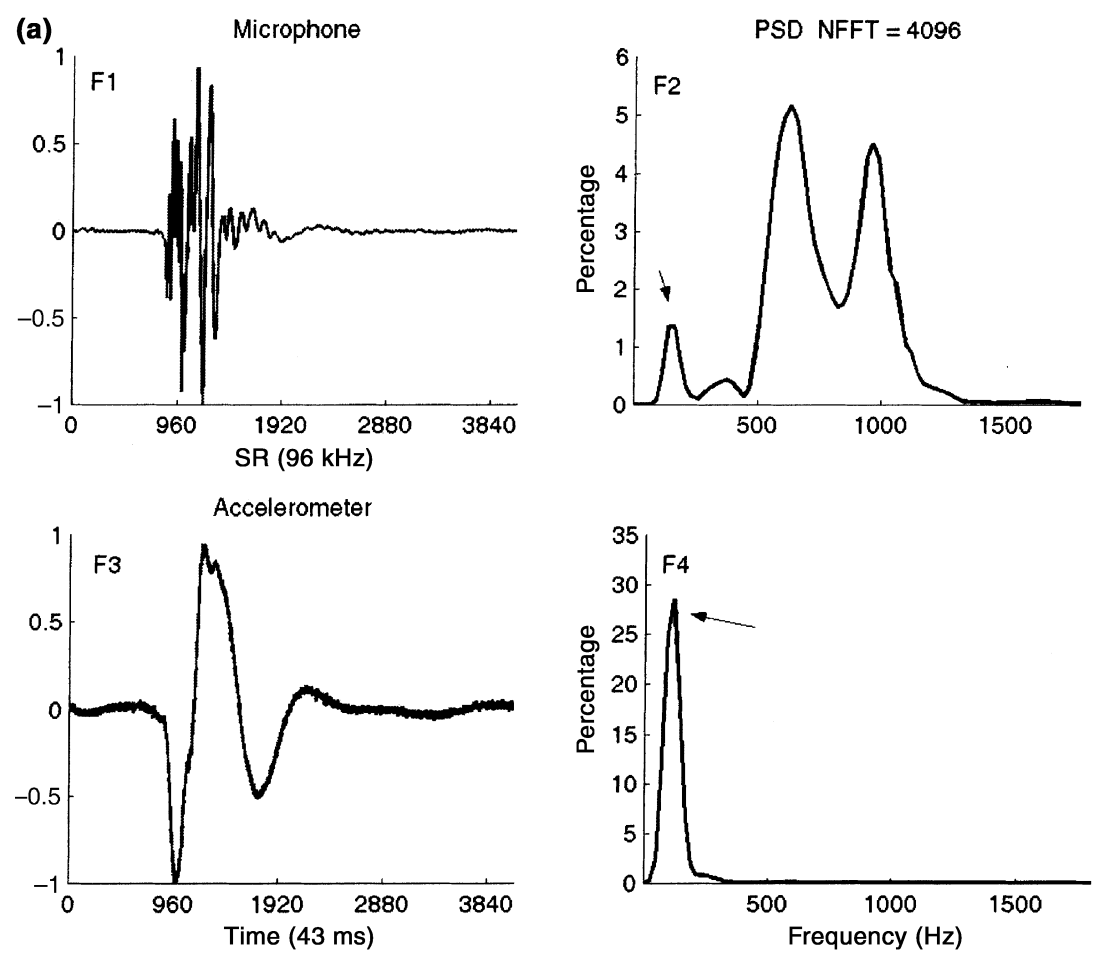

(b)

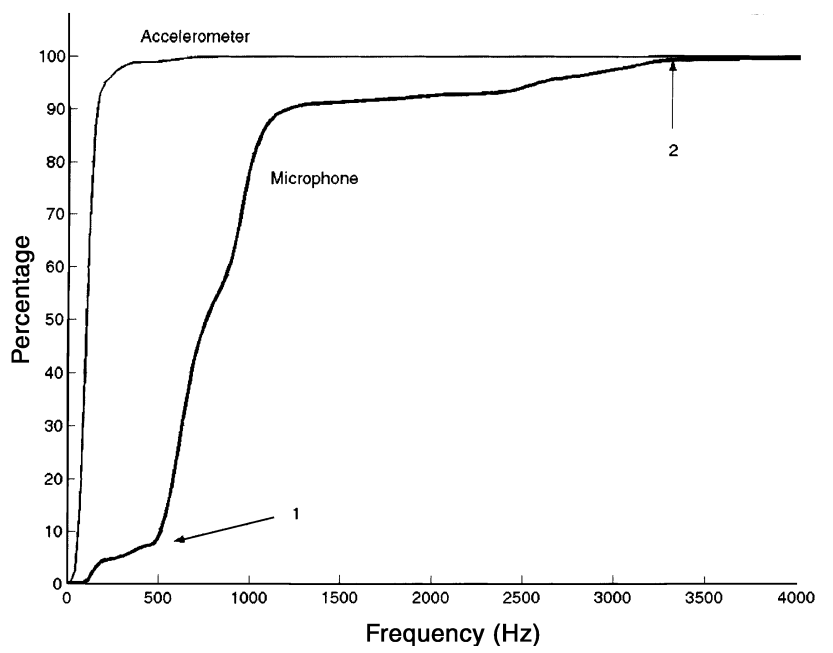

Fig. 4. (a) This figure illustrates the differences in the distribution of energy in relation to frequency between a microphone and a skin contact accelerometer recording. The TMJ sound recordings made with a microphone placed in the ear canal (analogue waveform in F1) at the side of the clicking TMJ and an accelerometer (analogue waveform in F3) placed in contact with the skin over the same side TMJ. Their power spectral densities (PSDs) are to the right of the analogue signals (windows F2, F4). Both transducers recorded the low frequency vibrations (arrows point at corresponding peaks) but the microphone also recorded large amounts of energy above $500 \mathrm{~Hz}$. That part of the signal is lost in the skin contact transducer recording. Sampling rate was $96000 \mathrm{~Hz}$. Vertical axes in Fl and F3 are in parts of the A/D card's dynamic range ( $1=$ maximal positive and $-1=$ maximal negative values). Vertical axes in F2 and F4 are in percentage of the signals' total energy amount. NFFT = length of the Fourier transform. (b) This figure illustrates the principal difference in results obtained by microphone versus skin contact accelerometer recordings of TMJ sounds. The graphs show the difference in cumulative energy distribution of the clicking in Fig. 4a recorded with a microphone (thick line) and with a skin contact accelerometer (thin line). The vertical axis is labelled in percentage of total energy. In the accelerometer recording almost $100 \%$ of the energy is below $300 \mathrm{~Hz}$ while more than $90 \%$ of the energy is above $500 \mathrm{~Hz}$ in the microphone recording (arrow 1). Note that the microphone recorded TMJ clicking has about $10 \%$ of its energy in the area from about $1200 \mathrm{~Hz}$ up to about $3300 \mathrm{~Hz}$ (arrow 2). It is obvious that huge amounts of energy above $300 \mathrm{~Hz}$ was lost and not recorded when using the skin contact accelerometer. 

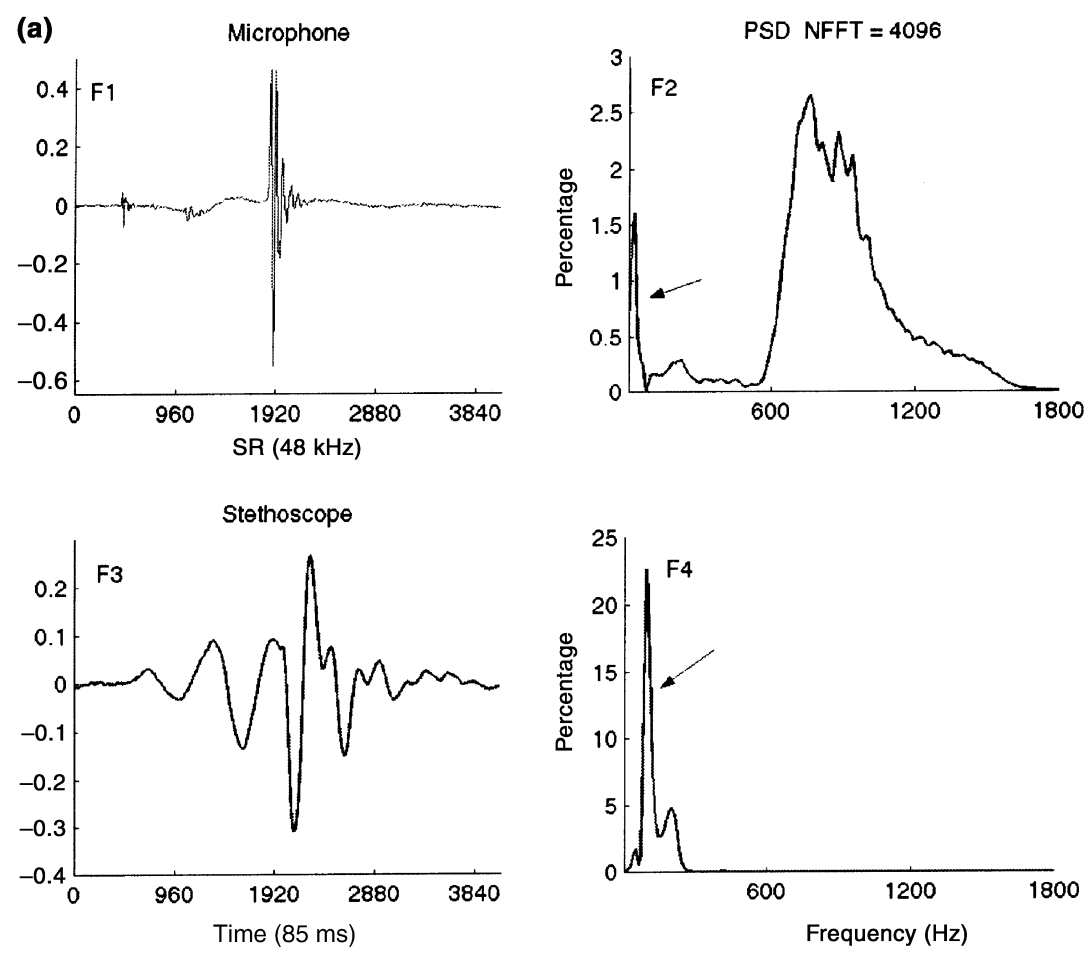

(b)

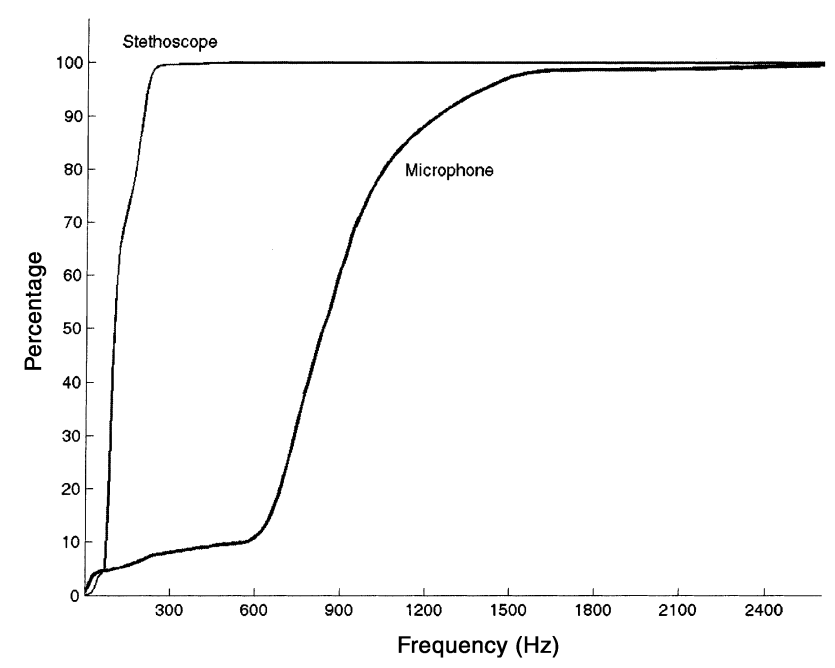

Fig. 5. (a) The same clicking was recorded with a microphone placed in the ear canal (F1) and with a microphone placed in the earplug of a stethoscope held in contact with the skin above the same side TMJ (F3). Note the 'smoothed out' appearance of the stethoscope recording that indicates a stronger dominance of low frequencies as compared with the more peaky microphone signal. The power spectral densities (PSDs) are in windows F2, F4 to the right of the analogue signals. Note that most energy in the spectrum of the microphone recording is above $600 \mathrm{~Hz}$ while most energy in the stethoscope recording spectrum is below $300 \mathrm{~Hz}$. Vertical axes in Fl and F3 are in parts of the A/D card's dynamic range ( $1=$ maximal positive and $-1=$ maximal negative values). Vertical axes in F2 and F4 are in percentage of the signals' total energy amount. The arrows point at corresponding peaks in the power spectra. NFFT $=$ length of the Fourier transform. (b) The graphs show the difference in cumulative energy distribution of the TMJ sound in Fig. 5a as recorded with a microphone in the ear canal (thick line) and with a microphone placed in the earpiece of a stethoscope (thin line) that was held with skin contact above the clicking joint. The vertical axis is labelled in percentage of total energy. In the stethoscope recording almost $100 \%$ of the energy is below $300 \mathrm{~Hz}$ while most of the energy, about 90\%, is above $600 \mathrm{~Hz}$ in the microphone recording. Note that the TMJ clicking has a clearly visible part of its energy in the area above $1500 \mathrm{~Hz}$. It is obvious that huge amounts of energy above $300 \mathrm{~Hz}$ were lost and not recorded when using a stethoscope. 

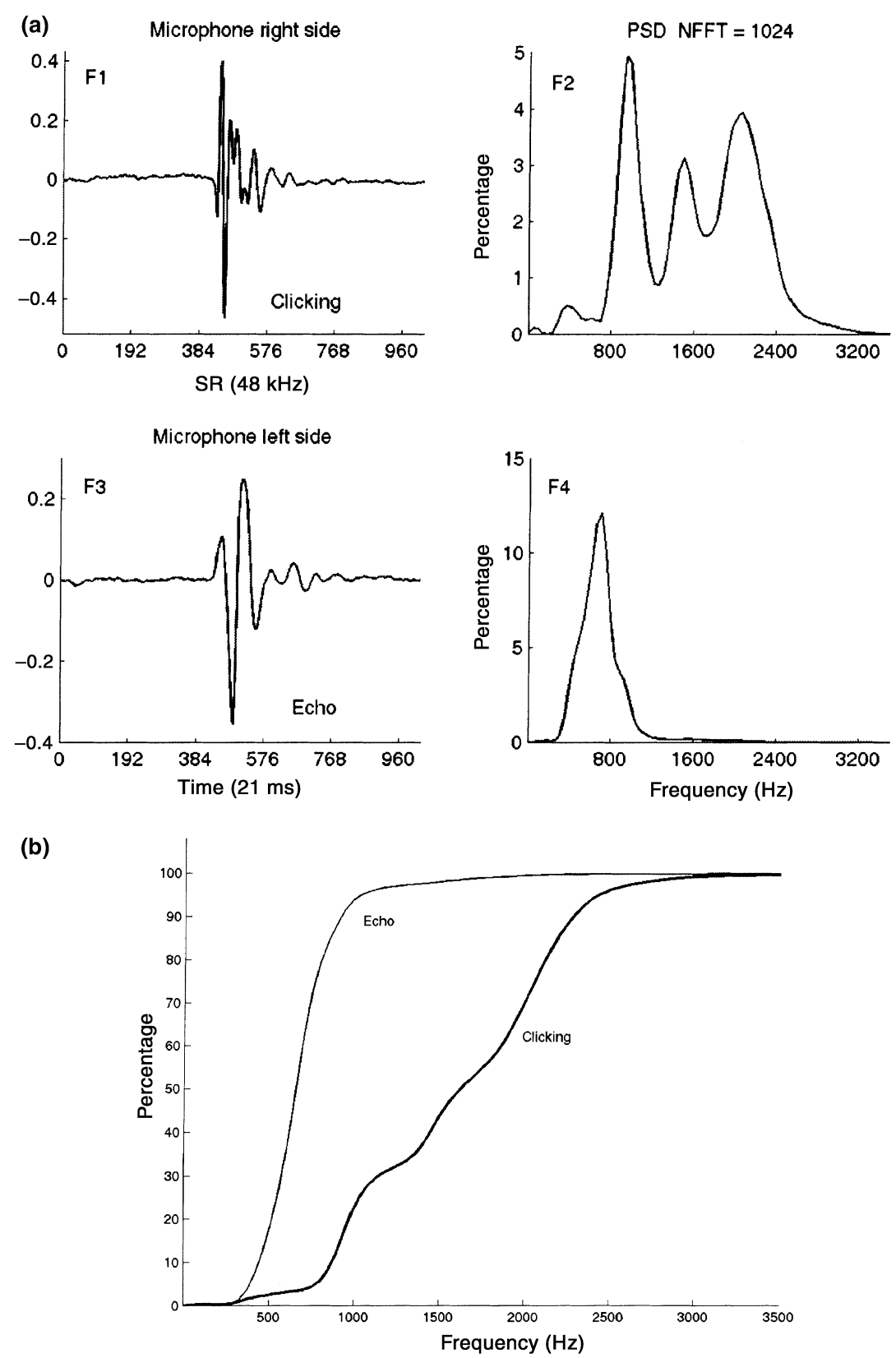

Fig. 6. (a) The TMJ clicking (F1) from the right TMJ was recorded with a microphone in the right ear canal. The 'echo' (F3) was recorded with a microphone placed in the left side ear canal. The power spectral densities (PSDs) are in windows F2, F4 to the right of the analogue signals. Note the differences in shape of waveforms and spectra. The clicking is more peaky than the echo that is 'smoothed out'. The clicking energy is distributed over a much higher frequency range than that of the echo. The differences are most probably due to the filtering effect of the head tissues. The echo has a longer distance to pass through head tissues before reaching the microphone. Vertical axes in $\mathrm{Fl}$ and $\mathrm{F} 3$ are in parts of the A/D card's dynamic range ( $1=$ maximal positive and $-1=$ maximal negative values). Vertical axes in F2 and F4 are in percentage of the signals' total energy amount. NFFT = length of the Fourier Transform. (b) This figure illustrates how the energy content of the sound signals is dependent on how much tissue the signals have to pass through. The graphs show the difference in cumulative energy distribution of the clicking in Fig. 6 a as recorded with a microphone on the clicking side (thick line) and the echo recorded with a microphone placed in the ear canal opposite to the clicking joint (thin line). The vertical axis is labelled in percentage of total energy. In the echo recording most of the energy is below $800 \mathrm{~Hz}$ while most of the energy $(\sim 90 \%)$ is above $800 \mathrm{~Hz}$ in the recording with the microphone on the clicking side. This indicates that energy is lost, especially in the high frequency areas, during passage through head tissues. Such loss should also affect comparisons between skin contact recordings from subjects with different thickness of adipose tissue in the TMJ areas. 
Table 1. Paired $t$-test

\begin{tabular}{|c|c|c|c|c|c|}
\hline & \multirow[b]{2}{*}{$n$} & \multicolumn{3}{|c|}{ Energy peak location } & \multirow[b]{2}{*}{$P$-value } \\
\hline & & $\begin{array}{l}\text { Session } 1 \\
\text { Mean } \pm \text { s.e. }\end{array}$ & $\begin{array}{l}\text { Session } 2 \\
\text { Mean } \pm \text { s.e. }\end{array}$ & $\begin{array}{l}\text { Session } 3 \\
\text { Mean } \pm \text { s.e. }\end{array}$ & \\
\hline \multicolumn{6}{|l|}{ Subject no. 1} \\
\hline Microphone in ear & & $443 \pm 51 \cdot 4$ & $548 \pm 43 \cdot 7$ & $544 \pm 16 \cdot 9$ & \\
\hline Accelerometer & 15 & $150 \pm 5 \cdot 0$ & & & $<0.001$ \\
\hline Stethoscope & 20 & & $235 \pm 19 \cdot 8$ & & $<0.001$ \\
\hline Microphone echo & 25 & & & $454 \pm 29 \cdot 0$ & $<0.01$ \\
\hline \multicolumn{6}{|l|}{ Subject no. 2} \\
\hline Microphone in ear & & $236 \pm 6 \cdot 2$ & $211 \pm 24 \cdot 4$ & $268 \pm 14 \cdot 9$ & \\
\hline Accelerometer & 15 & $97 \pm 2 \cdot 1$ & & & $<0.001$ \\
\hline Stethoscope & 13 & & $153 \pm 3 \cdot 3$ & & $<0.05$ \\
\hline Microphone echo & 21 & & & $181 \pm 8 \cdot 1$ & $<0.001$ \\
\hline \multicolumn{6}{|l|}{ Subject no. 3} \\
\hline Microphone & & $1366 \pm 39 \cdot 3$ & $845 \pm 8 \cdot 9$ & $1791 \pm 11 \cdot 2$ & \\
\hline Accelerometer & 15 & $81 \pm 7 \cdot 2$ & & & $<0.001$ \\
\hline Stethoscope & 10 & & $141 \pm 5 \cdot 2$ & & $<0.001$ \\
\hline Microphone echo & 14 & & & $670 \pm 6 \cdot 5$ & $<0.001$ \\
\hline Total $n$ & 148 & & & & \\
\hline
\end{tabular}

Values are in $\mathrm{Hz}$.

Mean (in $\mathrm{Hz}$ ) and standard errors (s.e.) are given for each recording session from subjects nos 1-3. Microphone in ear = recording from a microphone placed in the ear canal at the same side as the clicking joint. Accelerometer = accelerometer held in place by a headphone holder on the skin above the clicking joint. Stethoscope $=$ microphone in earplug of a stethoscope with the end-piece held in contact with the skin above the clicking joint. Microphone echo = microphone placed in the ear canal on the side opposite to the clicking joint. $n=$ number of two-channel recordings. $P=$ probability.

The ranges of the energy peak locations were for the recordings with the microphone in the ear canal at the clicking side $117-1922 \mathrm{~Hz}(n=172)$; for the microphone at the side opposite to the clicking joint 141$703 \mathrm{~Hz}(n=60)$; for the skin contact accelerometer $47-188 \mathrm{~Hz}(n=45)$; and for the stethoscope recording (with microphone in the ear plug) $125-375 \mathrm{~Hz}$ $(n=43)$ (Table 1).

The energy peaks were always in a significantly higher part $(P<0.001)$ of the frequency domain in the recordings with the microphone at the opening of the auditory canal than with the second microphone held either a few millimetres above the skin or in contact with the skin above the clicking joint (Table 2).

\section{Discussion}

Electronic TMJ sound recordings have the potential to be of diagnostic value. The results from such recordings may, however, be as unreliable as auscultatory findings if recordings are made with methods that fail to record, without distortion, the waveforms and the energy content over the sounds' whole dynamic and frequency ranges. The main result of this study was that TMJ sounds, contrary to a widespread opinion, may certainly contain significant amounts of energy in the frequency areas well above $650 \mathrm{~Hz}$ but that those parts are lost, or have their energy content significantly reduced, in recordings with skin contact transducers. We therefore strongly object to those who claim that all or almost all the energy of TMJ vibrations is always in the area below $650 \mathrm{~Hz}$. The possible occurrence of higher frequencies and their significance as characteristics of TMJ vibrations should thus not be ignored when choosing sensors and SRs. The reason for the absence of high frequencies in the data of some studies (Ishigaki et al., 1993; Owen, 1996; Olivieri et al., 1999; Garcia et al., 2000) is to us a combination of their exclusive use of skin conduct transducers and too low SRs.

The aim of this study was not to establish normative values for the energy distributions in different types of TMJ sounds. That will be the subject of future studies. However, when planning those it is necessary to first know if there is a systematic difference in spectra 
Table 2. Paired $t$-test

\begin{tabular}{|c|c|c|c|c|}
\hline & \multirow[b]{2}{*}{$n$} & \multicolumn{2}{|c|}{ Energy peak location } & \multirow[b]{2}{*}{$P$-value } \\
\hline & & $\begin{array}{l}\text { Session } 4 \\
\text { Mean } \pm \text { s.e. }\end{array}$ & $\begin{array}{l}\text { Session } 5 \\
\text { Mean } \pm \text { s.e. }\end{array}$ & \\
\hline \multicolumn{5}{|l|}{ Subject no. 1} \\
\hline Microphone in ear & & $533 \pm 141 \cdot 3$ & $592 \pm 29 \cdot 3$ & \\
\hline Second microphone about $2 \mathrm{~mm}$ above the TMJ skin & 11 & $230 \pm 22 \cdot 3$ & & $<0.001$ \\
\hline Second microphone in contact with skin above TMJ & 13 & & $174 \pm 6 \cdot 3$ & $<0.001$ \\
\hline Total $n$ & 24 & & & \\
\hline
\end{tabular}

Values are in $\mathrm{Hz}$.

Mean (in $\mathrm{Hz}$ ) and standard errors (s.e.) of energy peak locations are given for recording sessions 4-5 from subject no. 1. Microphone in ear = recording from a microphone placed in the ear canal at the same side as the clicking joint. Second microphone was, in session 4 , held about $2 \mathrm{~mm}$ above the skin above the clicking TMJ. In session 5 the second microphone was held in contact with the skin above the clicking joint. $n=$ number of two-channel recordings. $P=$ probability. The energy peaks were located in significantly lower frequency areas in the recordings with the second microphone than in the recordings with the microphone placed in the ear canal.

obtained with the different sensors and SRs used in earlier research. Most important for us was to compare between recordings with microphones and recordings with transducers placed with skin contact above the joint. Because we always compared pairs of recordings of the same sounds between sensors we feel confident that microphones do pick up significant amounts of high frequency vibrations that are very much attenuated or even lost if recording with skin contact transducers or using SRs as low as 1000-1300 Hz.

It is still an open question what the proportions of low versus high frequencies are in the original TMJ sounds before they are filtered by the tissues surrounding the TMJ on the way to recording contact sensors or microphones. None of the methods published so far can give a perfect representation of the original frequency distribution. Future studies may need to use more than one transducer type. Contact sensors and microphones have their pros and cons and there is no reason to always exclude one type for the other. Whatever be the type used, the SR has to be very much higher than $1300 \mathrm{~Hz}$ to allow a representative display of the frequency content of the TMJ sounds (Widmalm et al., 1996b). High SR are also needed to record clickings with short duration (1-2 ms). Those clickings may be very loud but become severely distorted or not recorded at all if the SR is as low as $1000 \mathrm{~Hz}$ (Widmalm et al., 1996b). We used an SR of 48000 or $96000 \mathrm{~Hz}$. Even higher SR may be needed to localize a clicking to side because the time difference between a clicking and its contra-laterally recorded echo may be less than $1 \mathrm{~ms}$
(Widmalm, Williams, \& Yang, 1999; Widmalm et al., 2002).

Our results support the authors (Ouellette, 1974; Gay \& Bertolami, 1987; Gallo et al., 1993; Widmalm et al., 1996a; Prinz, 1998;) who also often found energy in areas above $650 \mathrm{~Hz}$. We consider it not possible to presently say for sure what the upper limit is for the frequency range of TMJ sounds. It may well be that the high frequency parts above $2000 \mathrm{~Hz}$, even as high as $16000 \mathrm{~Hz}$, have more clinical significance than the low frequency parts as indicated by reports about knee joint sounds (Chu et al., 1976a, b; Chu, Gradisar \& Mostardi, 1978). Determining the upper limit of the TMJ sounds' frequency range and the prevalence of different types of frequency distributions will be the subject of future studies.

Like most authors we also found large amounts of energy in low frequency areas well below $650 \mathrm{~Hz}$ in the microphone recordings (Oster et al., 1984; Remington et al., 1990; Tallents et al., 1993). This means that low frequency energy may be attenuated but not necessarily lost. The proportions of low versus high frequency energy in the power spectra were, however, very different in the contact sensor and the microphone recordings (Fig. 4-6). The energy peaks were always in a higher frequency area in the microphone recordings than in the simultaneously made contact sensor recordings.

There are several possible reasons for the differences in relative distribution of low versus high frequencies between the microphone and the contact sensor 
recordings. One is the loose attachment of the accelerometer when placed in contact with the skin above the TMJ. Accelerometers are supposed to be firmly attached to a hard surface to avoid losing parts of the signal's high frequency content. Such a mounting is possible when recording TMJ sounds only by fastening the accelerometers to the subject's teeth as Drum \& Litt (1987) did. Such a method is, however, not convenient for routine use in clinical applications, although in autopsy studies, the accelerometer can be fastened to the metal face of the holder where the specimen is fixed (Widmalm et al., 1992). When mounting the accelerometers on a hard surface Drum \& Litt (1987) and Widmalm et al. (1992) found the frequency range of TMJ sounds to extend far beyond $650 \mathrm{~Hz}$.

The main reason for using skin contact transducers is based on the theory that lower frequency sounds are best recorded with contact sensors. Groups advocating contact sensors (Frank, Rangayyan \& Bell, 1990; Owen, 1996; Bracco et al., 1997) often refer to Mollan, McCullagh \& Wilson (1982) and to Kernohan et al. (1990). They pointed out that when the TMJ sounds propagate through the skin barrier into air, part of the energy is reflected due to the difference in acoustic impedance between the emitting bone/skin system and the surrounding air. This affects the lower frequencies more than the higher, and the loss of low frequency energy is less if the vibrations are recorded with contact sensors before they get air-borne. It is, however, important to note that their concerns were primarily about subsonic vibrations. Depending on filter settings the lower limit may be about $30 \mathrm{~Hz}$ in the equipment most authors use today for TMJ sound/vibration recordings. As far as we can find only Drum \& Litt (1987) have recorded TMJ vibrations with equipment capable of picking up subsonic vibrations. They used B \& K miniature accelerometers (model $4374^{* * *}$ ), which had a flat frequency response range of DC to $50000 \mathrm{~Hz}$. They attached the accelerometer in the best possible way by having it rigidly fixed to a mandibular tooth. Drum \& Litt (1987) found, however, the lower end of the frequency range to be $50 \mathrm{~Hz}$ when recording from 50 subjects with a wide range of TMJ pathology. The significance of subsonic vibrations is thus not known because to the best of our knowledge no published

***Bruel and Kjaer, Marlborough, MA, U.S.A. studies have observed, or been able to record such low frequency vibrations from the TMJ.

The sounds have to pass through larger amounts of soft tissues before being recorded with contact sensors than before being recorded with a microphone in the ear canal at the same side as the clicking joint. Peura (1992) pointed out that sounds are attenuated when travelling through the body to the surface and that the largest attenuation occurs in the most compressible tissues, such as fat layers. The thickness of soft tissues, especially the adipose tissues, in the TMJ area where skin contact transducers are placed (Fig. 1) may vary significantly between subjects. Another advantage with using a microphone in the auditory meatus is therefore that the layers between the TMJ and the auditory canal are relatively thin (Fig. 1) and most probably less variable between subjects than in the facial areas where skin contact transducers are placed. Our results support earlier findings (Widmalm et al., 2002) that the length of the path through head tissues, that the sound has to travel, affects the energy distribution and that the high frequency parts lose relatively more energy than the low frequencies. In the microphone recordings the energy peaks were observed at lower frequencies (Fig. 6) in recordings from the contra lateral side than in recordings from the same side as the clicking joint. That result is also supported by a study on dry bone skulls (Stenfelt, Håkansson \& Tjellström, 2000) where it was found that the trans-cranial attenuation was frequency dependent with a tendency towards higher attenuation at the higher frequencies.

We did not observe any differences in noise levels between sensors when using them for recording. The accelerometer was less sensitive to external sounds and had a lower noise level than the microphones we used but only when tested before being placed for recording. When attached in contact with the skin the noise increased to the same level as that of the microphones. It is, however, important to make sure that all persons in the room are silent during the recording. The microphone will pick up audible external sounds also when the auditory canal is sealed.

Results of research about vibratography are sometimes presented in a confusing way. Olivieri et al. (1999), for instance, reported that they calculated and graphed the averages of the amount of vibratory energy measured in Hertz! They gave average values of 'Total integral $(\mathrm{Hz})^{\prime}$ in their Tables 1 and 2 with figures in the range $2 \cdot 3-9 \cdot 7 \mathrm{~Hz}$. The information is perplexing 
because energy cannot be measured in Hz. Even more baffling is their citation on page 177 where they wrote; ${ }^{\text {Brooks }}{ }^{26}$ observed that $100 \%$ of the patients with posttrauma articular inflammation, evaluated through electrovibratography, showed vibrations of low amplitude and lower frequencies of between $0-25 \mathrm{~Hz}^{\prime}$. The alleged reference (Brooks \& Westesson, 1993) does not include recording or analysis of TMJ sounds. Nor could we find any other paper reporting about electrovibratography with such results as claimed in the citation.

Gallo et al. (1993) used a microphone fitted into the earpieces of a stethoscope. Placing a small microphone in the earplug of the stethoscope does, however, not mean that the recorded sound is a true representation of what we hear when using a stethoscope in auscultation. The mechanical stethoscope amplifies sound because of a standing-wave phenomenon in the listener's ear canal (Peura, 1992). This means that the acoustic characteristics of the sound perceived by the listener may differ greatly from those of the sound electronically recorded to a computer via a microphone in the earpiece. Similar effects may influence the recordings by microphones placed in the ear canal and further studies are needed before discussing the possible implications. Nor does the microphone-stethoscope combination solve the problem with impedance shift. The diaphragm acts as a contact sensor but the vibrations picked up that way have still to pass an 'impedance-barrier' when entering the air in the tubing. It is also important to note that the recording characteristics may vary between stethoscopes (Ertel et al., 1966a, b) to such a degree that comparing between examiners becomes impossible. When a microphone is used it seems to us more convenient to place it directly into the ear canal.

In conclusion a microphone placed in the ear canal has the advantage of being as close as possible to the TMJ sound source. The microphone is superior to the skin contact transducers in recording frequencies above $300-650 \mathrm{~Hz}$ and those may be abundant in TMJ vibration recordings. There is no scientific reason to claim that only the vibrations below $650 \mathrm{~Hz}$ have diagnostic significance. Consequently there is no reason to deliberately exclude the frequencies above $650 \mathrm{~Hz}$ by only using contact sensors and/or by sampling with $1300 \mathrm{~Hz}$ or lower rates. Low frequency vibrations may be more attenuated in microphone than in skin contact transducer recordings but they are not missed. However, whatever type of transducer used for recording of
TMJ vibrations, high SRs are needed and we recommend $48000 \mathrm{~Hz}$ or higher. Further studies are needed to establish normative values for frequency ranges of normal joint sounds versus those correlated to pathological joint changes.

\section{References}

Ballou, G.M. (1998) Handbook for Sound Engineers, 2nd edn, p. 23. Focal Press, Boston.

Bracco, P., Deregibus, A., Piscetta, R. \& Giaretta, G.A. (1997) TMJ clicking: a comparison of clinical examination, sonography, and axiography. Journal of Craniomandibular Disorders, 15, 121.

Brooks, S.L. \& Westesson, P.L. (1993) Temporomandibular joint: value of coronal MR images. Radiology, 188, 317.

Chu, M.L., Gradisar, I.A., Railey, M.R. \& Bowling, G.F. (1976a) Detecting of knee joint diseases using acoustical pattern recognition technique. Journal of Biomechanics, 9, 111.

Chu, M.L., Gradisar, I.A., Railey, M.R. \& Bowling, G.F. (1976b) An electro-acoustical technique for the detection of knee joint noise. Medical Research Engineering, 12, 18.

Chu, M.L., Gradisar, I. \& Mostardi, R. (1978) A noninvasive electro-acoustical evaluation technique of cartilage damage in pathological knee joints. Medical and Biological Engineering and Computing, 16, 437.

Drum, R. \& LitT, M. (1987) Spectral analysis of temporomandibular joint sounds. Journal of Prosthetic Dentistry, 58, 485.

Ekensten, B. (1952) Phonograms of anomalies of the temporomandibular joint in motion. Odontologisk Tidskrift, 60, 235.

Ertel, P.Y., Lawrence, M., Brown, R.K. \& Stern, A.M. (1966a) Stethoscope acoustics. I. The doctor and his stethoscope. Circulation, 34, 889.

Ertel, P.Y., Lawrence, M., Brown, R.K. \& Stern, A.M. (1966b) Stethoscope acoustics. II. Transmission and filtration patterns. Circulation, 34, 899.

Frank, C.B., Rangayyan, R.M. \& Bell, G.D. (1990) Analysis of knee joint sound signals for non-invasive diagnosis of cartilage pathology. IEEE Engineering in Medicine and Biology Magazine, 9, 65 .

Gallo, L.M., Airoldi, R., Ernst, B. \& Palla, S. (1993) Power spectral analysis of temporomandibular joint sounds in asymptomatic subjects. Journal of Dental Research, 72, 871.

Garcia, A.R., Madeira, M.C., Paiva, G. \& Olivieri, A.N. (2000) Joint vibration analysis in patients with articular inflammation. Journal of Craniomandibular Practice, 18, 272.

Gay, T. \& Bertolami, C.N. (1987) The spectral properties of temporomandibular joint sounds. Journal of Dental Research, 66, 1189.

Hashimoto, K., Takehana, S., Ito, Y., Takenaka, M., Suzuki, N. $\delta$ Abe, T. (1990) A study of how to distinguish the side on which TMJ noise is occurring. Aichi Gakuin Daigaku Shigakkai ShiAichi-Gakuin Journal of Dental Science, 28, 1151.

Hutta, J.L., Morris, T.W., Katzberg, R.W., Tallents, R.H. $\delta$ EsPeland, M.A. (1987) Separation of internal derangements of the temporomandibular joint using sound analysis. Oral Surgery, Oral Medicine, Oral Pathology, 63, 151. 
Ishigaki, S., Bessette, R.W. \& Maruyama, T. (1993) A clinical study of temporomandibular joint vibrations in TMJ dysfunction patients. Journal of Craniomandibular Practice, 11, 7.

JARvis, C. (1992) Physical Examination and Health Assessment, p. 169. Saunders, Philadelphia, PA.

JeONG, J. \& Williams, W.J. (1992) Alias-free generalized discretetime time-frequency distributions. IEEE Transactions on Signal Processing, 40, 2757.

Kernohan, W.G., Beverland, D.E., McCoy, G.F., Hamilton, A., Watson, P. \& Mollan, R. (1990) Vibration arthrometry. A preview. Acta Orthopedica Scandinavica, 61, 70.

Leader, J.K., Boston, J.R., Rudy, T.E., Greco, C.M. \& Zaki, H.S. (1999) The influence of mandibular movements on joint sounds in patients with temporomandibular disorders. Journal of Prosthetic Dentistry, 81, 186.

Mendel, L.L., Danhauer, J.L. \& Singh, S.S. (1999) Singular's Illustrated Dictionary of Audiology, pp. 197, 224, 239. Singular Publishing Group, San Diego, CA.

Mollan, R.A.B., McCullagh, G.C. \& Wilson, R.I. (1982) A critical appraisal of auscultation of human joints. Clinical Orthopaedics and Related Research, 170, 231.

Norušs, M.J. (2000) Guide to Data Analysis, pp. 240. Prentice Hall, Englewood Cliffs, NJ.

Olivieri, K.A.N., Garcia, A.R., Paiva, G. \& Stevens, C. (1999) Joint vibration analysis in asymptomatic volunteers and symptomatic patients. Journal of Craniomandibular Practice, 17, 176.

Oster, C., Katzberg, R.W., Tallents, R.H., Morris, T.W., Bartholomew, J., Miller, T.L. \& Hayakawa, K. (1984) Characterization of temporomandibular joint sounds. A preliminary investigation with arthrographic correlation. Oral Surgery, Oral Medicine, Oral Pathology, 58, 10.

OuelLette, P.L. (1974) TMJ sound prints: electronic auscultation and sonographic audiospectral analysis of the temporomandibular joint. Journal of the American Dental Association, 89, 623.

Owen, A.H. (1996) Rationale and utilization of temporomandibular joint vibration analysis in an orthopedic practice. Journal of Craniomandibular Practice, 14, 139.

Peura, R.A. (1992) Blood Pressure and Sound. Medical Instrumentation. Application and Design (ed J.G. Webster), pp. 378. Houghton Mifflin Company, Boston.

PRINZ, J.F. (1998) Subjective assessment of temporomandibular joint sounds. Journal of Oral Rehabilitation, 25, 765.

Remington, K.J., SAdowsky, C., Muhl, Z.F. \& Begole, E.A. (1990) Timing and character of reciprocal temporomandibular joint sounds in an asymptomatic orthodontic sample. Journal of Craniomandibular Disorders: Facial and Oral Pain, 4, 21.

Sano, T., Widmalm, S.E., Westesson, P.L., Takahashi, K., Yoshida, H., Michi, К. \& Oкаno, T. (1999) Amplitude and frequency spectrum of TMJ sounds from subjects with and without other signs/symptoms of temporomandibular disorders. Journal of Oral Rehabilitation, 26, 145.
Stenfelt, S., HÅkansson, B. \& TJellström, A. (2000) Vibration characteristics of bone conducted sound in vitro. Journal of the Acoustical Society of America, 107, 422.

Tallents, R.H., Hatala, M., Katzberg, R.W. \& Westesson, P.L. (1993) Temporo mandibular joint sounds in asymptomatic volunteers. Journal of Prosthetic Dentistry, 69, 298.

WATt, D.M. (1963) A preliminary report on the auscultation of the masticatory mechanism. Dental Practitioner, 14, 270.

WATT, D.M. (1966) Gnathosonics - A study of sounds produced by the masticatory mechanism. Journal of Prosthetic Dentistry, 16, 73 .

WeLch, P.D. (1967) The use of Fast Fourier Transform for the estimation of power spectra: a method based on time averaging over short, modified periodograms. IEEE Transactions Audio Electroacoustical, AU-15, 70.

Widmalm, S.E. \& HedegÅrd, B. (1974) An apparatus for the synchronous registration of EMG activity in jaw muscles and of vibrations in the masticatory system. Journal of Oral Rehabilitation, 1, 183.

Widmalm, S.E., Williams, W.J. \& Zheng, C. (1991) Time frequency distributions of TMJ sounds. Journal of Oral Rehabilitation, 18, 403.

Widmalm, S.E., Westesson, P.L., Brooks, S.L., Hatala, M.P. \& PAesani, D. (1992) Temporomandibular joint sounds: correlation to joint morphology in fresh autopsy specimens. American Journal of Orthodontics and Dentofacial Orthopedics, 101, 60.

Widmalm, S.E., Williams, W.J., Christiansen, R.L., Gunn, S.M. $\delta$ PARK, D.K. (1996a) Classification of temporomandibular joint sounds based upon their reduced interference distribution (RID). Journal of Oral Rehabilitation, 23, 35.

Widmalm, S.E., Williams, W.J. \& Adams, B.S. (1996b) The wave forms of temporomandibular joint sounds clicking and crepitation. Journal of Oral Rehabilitation, 23, 44.

Widmalm, S.E., Williams, W.J. \& YANG, K.P. (1999) False localization of TMJ sounds to side is an important source of error in TMD diagnosis. Journal of Oral Rehabilitation, 26, 213.

Widmalm, S.E., Williams, W.J. \& McKay, D.C. (2002) Localization of TMJ sounds to side. Journal of Oral Rehabilitation, 29, 911-917.

Williams, W.J. \& JeONG, J. (1991) Reduced interference timefrequency distributions. In: Time-Frequency Signal Analysis: Methods and Applications, Chapter 3 (ed. B. BoAshash). Longman Cheshire, Melbourne, Australia.

Yoshida, H., Sano, T., Kataoka, R., Takahashi, K. \& Michi, K. (1994) A preliminary investigation of a method of detecting temporomandibular joint sounds. Journal of Orofacial Pain, 8, 73 .

Correspondence: Sven E Widmalm, 1565 Kuehnle, Ann Arbor, MI 48103, U.S.A.

E-mail: sew@umich.edu 